\title{
Salus et fides. Secularización y política mesiánica en La Estrella de la Redención
}

Resumen: Este trabajo ofrece un estudio del análisis teológico-político del Estado nacional moderno propuesto por Franz Rosenzweig en La Estrella de la Redención. Se persigue destacar la atención que Rosenzweig prestó al problema de la secularización y, por tanto, a la teología política, entendida ésta, en La Estrella, como "política mesiánica": cristianización de "los pueblos del mundo" como condición del auge del nacionalismo que condujo a Europa hacia la mundialización de la "guerra de religión" (Primera Guerra Mundial), es decir, hacia una guerra mundial inter-nacional por la unidad de salus (auto-conservación nacional) y fides (fidelidad a Dios). De este modo se sientan las bases para una lectura teológico-política de la tercera parte de La Estrella, que de acuerdo con la hipótesis de trabajo del presente estudio debe ser entendida como una respuesta a la teoría hegeliana de la mundanización de la religión (cristiana) a través del Estado (racional). Ésta será objeto de exposición en la primera parte del artículo. A continuación expondré el análisis de la articulación hegeliana de política, historia y religión que cabe encontrar en el Hegel de Rosenzweig y, por último, la decisivia función que desempeña en La Estrella.

Palabras clave: política mesiánica, secularización, teología política, salus, fides

Abstract: This paper deals with the theological-political interpretation of modern national State presented by Franz Rosenzweig in The Star of Redemption. I will try to emphasize the attention that Rosenzweig paid to secularization problem and therefore to political theology, which the author of The Star denominates "messianic politics": christianization of "the peoples of the world" as condition of the rise of nationalism which led to mundialization of "religious war" (First World War), i. e. to inter-national world war in name of the unity of salus (national self-preservation) and

* Doctor Internacional en Filosofía por la Universidad Autónoma de Madrid (2013). Ha sido becario de investigación de dicha universidad, así como del Servicio Alemán de Intercambio Académico (DAAD). Entre sus publicaciones, destaca la co-edición, junto a Valerio Rocco Lozano, de Teología y teonomía de la política (Abada, Madrid, 2012), así como la traducción y edición de los escritos políticos de Franz Rosenzweig, a aparecer próximamente en la editorial Sígueme (Salamanca), bajo el título Globus y otros escritos sobre la guerra. Dirección electrónica: roberto.navarrete@uam.es 
fides (faithfulness to God). This way I will set the basis for a theological-political reading of the Third Part of The Star, which according to the hypothesis of the present essay is to be understood as a response to Hegel's theory of mundanization of (christian) religion through (rational) State. This Hegelian doctrine will be presented in the first section of the paper. Next I will expound Rosenzweig's early analysis of Hegel's articulation between politics, history and religion and, finally, the decisive role which subsequently he plays in The Star.

Keywords: messianic politics, secularization, political theology, salus, fides.

I.

Bajo el título "Política mesiánica", Franz Rosenzweig expone en $\mathrm{La}$ Estrella de la Redención (1921) su particular interpretación de la relación moderna entre mesianismo y nacionalismo, es decir, su propia teoría de la secularización, que como tal debe ser estudiada en el marco del problema teológico-político. ${ }^{1}$ Pocos son, no obstante, los intérpretes de Rosenzweig que han destacado este aspecto de la tercera parte de su opus magnum, habitualmente interpretado en un sentido a-político, cuando no decididamente anti-político: a la manera de un ejemplo de teología judía, a lo sumo de filosofía judía de la religión, desprendida en cualquier caso de todo interés por el mundo, la historia y, por ende, la política ${ }^{2}$. Nada más lejos de la realidad. La tercera parte de la obra

${ }^{1}$ Cf. Rosenzweig, F., La Estrella de la Redención [EdR], tr. M. García-Baró, Sígueme, Salamanca, 1997, pp. 389ss.

2 Este tipo de recepción estuvo determinada por la extraordinariamente influyente biografía intelectual de Rosenzweig elaborada por Nahum N. Glatzer: Franz Rosenzweig. His life and thought, Schocken, New York, $1961^{2}\left(1953^{1}\right)$. Entre las interpretaciones de Rosenzweig en clave apolítica destacan: Freund, E.-R., Die Existenzphilosophie Franz Rosenzweigs: ein Beitrag zur Analyse seines Werkes Der Stern der Erlösung, Meiner, Hamburg, 1959, y Mayer, R., Fran₹ Rosen₹weig. Eine Philosophie der dialogischen Erfahrung, Chr. Kaiser Verlag, München, 1973, así como Casper, B., Das Dialogische Denken: Eine Untersuchung der religionsphilosophischen Bedeutung Franz. Rosenzweigs, Ferdinand Ebners und Martin Buber, Herder, Freiburg, 1967. Bernhard Casper, uno de los más lúcidos intérpretes del pensamiento rosenzweiguiano y una referencia constante para quien escribe, ha llamado la atención posteriormente sobre la dimensión (teológico-)política del Nuevo Pensamiento de Rosenzweig, por 
capital de Rosenzweig ofrece tanto una interpretación de la relación entre nacionalismo y mesianismo (que bien podría ser catalogada de "teoría de la secularización") como una alternativa a dicha teología política que, por su parte, debe ser entendida como una "nueva secularización"3 (del mesianismo o, mejor, dada la aversión de Rosenzweig por todo "-ismo", de la idea mesiánica). El presente estudio se ceñirá al primero de estos dos aspectos. Lo que de este modo se persigue es que el último representante del judaísmo alemán sea considerado, con pleno derecho, un pensador de la secularización: que La Estrella, siquiera la tercera de sus partes, sea leída en perspectiva teológico-política.

$\mathrm{Ha}$ de destacarse, en primer lugar, el origen hegeliano de la interpretación de la relación moderna entre religión y política presentada por Rosenzweig: aquellos pasajes de la Enciclopedia (\$552), como de la Filosofía del Derecho (\$270), en los que Hegel tematiza la relación entre religión y Estado desde el punto de vista de la historia universal, esto es, su tratamiento del cristianismo en el marco de la articulación de filosofía política y filosofía de la historia característica del sistema hegeliano. Hegel acepta la idea de una neutralidad religiosa del Estado moderno y, por lo tanto, la idea de tolerancia, es decir, la separación institucional de Iglesia y Estado resultante de la Reforma, que permite que las creencias religiosas de los individuos resulten indiferentes cuando de reconocer su personalidad jurídica se trata. Las relaciones del Estado con la religión, sin embargo, no se agotan en el plano del derecho positivo (la igualdad de todas las religiones ante la ley) sino que alcanzan el plano especulativo.

Para Hegel no es suficiente con dar al César lo que es del César y a Dios lo que es de Dios: debe determinarse conceptualmente aquello que corresponde a la religión y al Estado como figuras del Espíritu. Desde este punto de vista, no hay en Hegel una separación entre lo religioso y lo político.

ejemplo, en "Der Andere, der Dritte und die Bürgschaft für die Gerechtigkeit", en Archivio di Filosofia, LXXIV 1-3, 2006, 185-194.

${ }^{3}$ Cf. Rosenzweig, F., Der Mensch und sein Werk. Gesammelte Schriften (I): Briefe und Tagebücher 1 (1900-1918) [GSI], Martinus Nijhoff, Haag, 1979, vol. 2, pp. 888-890. 
Leemos, al contrario, que la religión es el basamento [Grundlage] del Estado. ${ }^{4}$ El derecho del Estado racional no radica en sí mismo, sino en el contenido de la religión y, de hecho, en el contenido de una religión: el luteranismo. El Estado es la realización objetiva de la misma verdad que la religión luterana alcanza sólo subjetivamente (en el plano de la representación y el sentimiento), es decir, la realización histórica del Espíritu en el mundo: su mundanización (Verweltlichung). Existe por tanto una unidad esencial entre religión (luterana) y Estado (ético o racional): en tanto que realización de la eticidad, él es realización de la religión en la que esta eticidad, como por lo tanto también el propio Estado, encuentra su sustancialidad (Substantialität) y su base $\left(\right.$ Basis). ${ }^{5}$

Verweltlichung se refiere en Hegel a la realización del cristianismo a través de una espiritualización de lo profano, una transformación del mundo por los principios espirituales del cristianismo y de éstos, a su vez, por el mundo. El movimiento dialéctico del que la Modernidad es resultado consistiría así en una mundanización de lo sagrado y una sacralización de lo temporal en la que el Estado aparece como instancia de mediación. ${ }^{6}$ Sin ser geistlich (eclesiástico), es geistig (espiritual): la Iglesia no posee el monopolio de esto espiritual y el Estado -al menos el Estado racional o ético- no es simplemente una institución secular, sino la realización del Espíritu en el mundo.

II.

$\mathrm{Al}$ escribir La Estrella, atrincherado en el frente balcánico de la Primera Guerra Mundial, Rosenzweig era perfectamente consciente del gozne cristiano que fundamentaba la articulación hegeliana de "filosofía de la historia" y "filosofía del Estado". Lo había estudiado en profundidad, antes

${ }^{4}$ Hegel, G. W. F., Principios de la Filosofía del Derecho o Derecho Natural y Ciencia Política, tr. J. L. Vermal, Edhasa, Barcelona, 1988, \270, pp. 335ss.

${ }^{5}$ Cf. Hegel, G. W. F., Enciclopedia de las ciencias filosóficas en compendio, tr. R. Valls Plana, Alianza, Madrid, 1997, \552, pp. 572ss.

${ }^{6}$ Cf. Rivera García, A., "La secularización después de Blumenberg", Res Publica, 1112, 2003, pp. 97-98. 
de 1914, de la mano del gran historiador alemán Friedrich Meinecke, primero con vistas a la obtención del doctorado y, después, en aras de una habilitación que nunca llegaría a consumarse -por expreso deseo de Rosenzweig, que había tomado ya la decisión de profundizar en el estudio del judaísmo al margen de la academia stricto sensi ${ }^{7}$ - Rosenzweig publicó su investigación una vez acontecida la primera catástrofe europea del siglo XX, en 1920, bajo el título Hegel und der Staat , añadiendo a lo concluido antes de 1914 un Prólogo y unas Consideraciones finales relativas al camino que condujo de Hegel a Bismarck y, después, del Canciller de Hierro a la guerra mundial. Son también el último testimonio de Rosenzweig como historiador (habilitable). Para entonces se había convertido en filósofo (aunque en uno absolutamente no habilitable): el autor de La Estrella de la Redención. Pero no nos llamemos a engaño: la sección de La Estrella dedicada a la messianische Politik tiene su origen, efectivamente, en la derrota de Alemania y el definitivo retorno de Rosenzweig al judaísmo, pero también -no debe ser esto olvidado- en el estudio de la metafísica hegeliana del Estado, que de hecho había encontrado en la catástrofe de Europa, según la interpretación rosenzweiguiana, su propia verificación.

El Hegel de Rosenzweig ofrece al lector una exposición de la génesis y el devenir de la idea hegeliana de Estado -primero, a través de las estaciones vitales (Lebensstationen) recorridas por Hegel entre Stuttgart (1770) y Jena (1804); después, sobrepasada la mitad de la vida del filósofo suabo, conforme a la ruta trazada por la fenomenología hacia la filosofía de la historia: transitando las épocas del mundo (Weltepochen) que transcurren de Napoleón (1806) a la Revolución de Julio (1830)-. La importancia, en este contexto, de las relaciones entre Iglesia -religión, más bien-y Estado - poder- es señalada por Rosenzweig desde el primer momento: Die Kirche, das Reich, der Staat und das Volk es, en conexión con el dístico hegeliano sobre la racionalidad de lo realmente efectivo, y viceversa, el tema con el que el Hegel es inaugurado por su autor. Éste presenta el sistema hegeliano como consumación de la síntesis de poder (Macht) y derecho (Recht), kratos y ethos o, como lo llamará Rosenzweig más tarde, haciéndose eco de una reflexión ciceroniana sobre la

7 Cf. GSI, 2, 679 .

8 Cf. Rosenzweig, F., Hegel und der Staat [HuS], Suhrkamp, Berlin, $2010^{3}$ (MünchenBerlin, 19201). 
res publica ulteriormente criticada por Agustín de Hipona en De Civitate Dei, salus et fides: Maquiavelo y Lutero9. Tal es en muy buena medida el hilo conductor de la exploración de las alturas del Kulturprotestantismus llevada a cabo por el discípulo de Meinecke, es decir, uno muy similar al que el maestro había seguido en su Weltbürgertum und Nationalstaat (1908), fuente de inspiración última del trabajo de Rosenzweig ${ }^{10}$.

La cuestión sale a relucir de modo eminente en el apartado dedicado por Rosenzweig a Jena, es decir, al momento en el que la doctrina hegeliana del Estado adquiere carácter sistemático y se integra, por tanto, en una filosofía de la historia universal: el Estado compite entonces con la Iglesia por la condición de "destino" y el enfrentamiento entre ambas instituciones pasa a responder a una necesidad histórica interna propia de la Edad Moderna. Tras la unidad de ambas en Grecia y el sometimiento del Estado a la Iglesia en el Medioevo (catolicismo), el principio del Estado moderno se cifra en su independencia con respecto a la Iglesia, esto es, en su secularización (Säkularisierung). Hegel no emplea, no obstante, este término jurídico, dotado de obvias connotaciones anticristianas y, de hecho, en buena medida antigermánicas: entre 1555 (firma de la Paz de Augburgo, o Paz de las Religiones) y 1803 (promulgación de la Reichsdeputationshauptschluss por la que el Reich secularizó sus principados eclesiásticos, salvo tres de ellos, para terminar disolviéndose en 1806) "secularización” y "destrucción del (no en vano) Sacro Imperio Romano Germánico" fueron prácticamente sinónimos.

En correspondencia con la distinción entre geistig y geistlich, a la que ya hemos aludido, Welt no se identifica en Hegel con el saeculum católicomedieval (tan ungeistig como entkirchlicht, al menos desde la querella de las investiduras), ni obviamente tampoco con la Natur de la bella mitología del paganismo que el catolicismo había desdivinizado. Welt es más bien el mundo de la religión del futuro hacia la que conduce la última figura histórica del cristianismo: el protestantismo. La doctrina de Lutero consuma, en definitiva,

${ }^{9}$ Cf. GSI, 1, 282-283, EdR, 391 y Rosenzweig, F., "Paralipomena", en Id., El país de los dos ríos. El judaísmo más allá del tiempo y la historia, tr. I. Ortega, Encuentro, Madrid, 2014, pp. 287ss.

${ }^{10}$ Cf. Meinecke, F., Weltbürgertum und Nationalstaat: Studien zur Genesis des deutschen Nationalstaates, Oldenbourg, München-Berlin, 1908. 
el destino histórico de la religión cristiana: la reconciliación de Estado y Reino de los Cielos en el plano del pensamiento, esto es, sólo a nivel de la conciencia. Únicamente de este modo, es decir mediante la consumación (Voll-endung) del cristianismo, señala Rosenzwieg interpretando a Hegel, es posible el Estado (racional o ético), del mismo modo que el cristianismo, como religión consumada, es sólo posible en la medida en que produce efectos en un Estado tal. Los dos destinos coincidirían así en uno de mayor altura.

He aquí, conforme a la reconstrucción rosenzweiguiana, el hegeliano Estado del futuro: la tercera edad del mundo, aquella hacia la que se dirige el Espíritu, a lomos del caballo de Napoleón, en dirección a la tierra de promisión que habita el nuevo pueblo elegido por la Historia Universal para ser portador suyo y, más aún, sede de la reconciliación final del Espíritu con el mundo. De las etapas de la vida, o si se prefiere de la ciencia de la experiencia de la conciencia, de la Fenomenología que es (sólo) la Introducción al Sistema, Hegel ha dado el salto a las épocas del mundo, al curso de los desarrollos históricos y, en particular, al comienzo de la época cristianogermánica, germano-protestante más bien, en la que el Estado puede ser concebido como realización de la eticidad (racionalidad). Esta articulación de filosofía política y filosofía de la historia alcanzará su punto culminante, como ya se señaló, en los Principios de la Filosofía del Derecho (1820) y en la Enciclopedia (1827). La metafísica hegeliana del Estado, tanto como el radical desprecio de Hegel hacia el catolicismo, alcanzan aquí carácter plenamente sistemático, conforme al célebre dictum hegeliano: "Lo que es racional es real, y lo que es real es racional". ${ }^{11}$ Con un matiz nada despreciable: no desde el principio -a pesar de que la razón borre el tiempo a su paso por la historia- sino sólo desde que "el cristianismo, por medio de la idea de un Reino de Dios sobre la Tierra, se convirtió en exigencia ética y en pauta de toda institución

${ }^{11}$ Hegel, G. W. F., Principios de la Filosofía del Derecho o Derecho Natural y Ciencia Política, p. 51. Cf. HuS, 355ss. Ha de señalarse, no obstante, que esta "identidad" (se trata más bien de un constante bucle de retroalimentación entre lo real y lo racional) es más bien sólo supuesta, más aún si se atribuye al Estado (que en Hegel no alcanza la esfera del Espíritu Absoluto, como tampoco del concepto). La verdad especulativa que anuncia el dístico de Hegel sólo es aplicable, en sentido estricto, a Dios. Cf. Hegel, G. W. F., Enciclopedia de las ciencias filosóficas en compendio, \$6, pp. 105ss. 
humana"12; en primer lugar, del Estado como portador de la Historia Universal: en tanto que individuo, o "Estado entre Estados", 13 sometido al tribunal universal de la Historia. Su condición histórico-universal es por tanto juzgada a través de su relación con el resto de unidades políticas, lo cual ocurre eminentemente a través de la guerra: "un pueblo es 'juzgado' por la historia en la medida en que es aniquilado o desplazado por otro" $14-\mathrm{y}$, por cierto, sin saberlo él mismo, sino en virtud del providencial secreto de la propia historia universal: la astucia de la razón ${ }^{15}$.

No por ello ha desaparecido de escena, sin embargo, la Iglesia, ni por lo tanto la religión o, mejor aún, la "comunidad eclesial" (Kirchengemeinde). La posibilidad del conflicto sigue entonces abierta. Se trata, en lo fundamental, de un conflicto doctrinal -"el Estado también tiene una doctrina"-16 entre Estado y Kirchengemeinde que Hegel, en un gesto -teológico-políticonuevamente determinado por el protestantismo, acaso por su protestantismo, resuelve concibiendo estas dos realidades como manifestaciones diversas de un mismo contenido espiritual: la religión en tanto que fuente de la conciencia subjetiva de la libertad y de la moralidad; el Estado, en tanto que figura efectiva (objetiva) y organización de un mundo. No se trata, ni mucho menos, de la unidad de Estado e Iglesia añorada por los románticos y la Restauración. La lucha contra la secularización en el sentido anticristiano del término no se libra, en el caso de Hegel, por medio de una eclesialización del

${ }^{12} \mathrm{HuS}, 355$.

${ }_{13} \mathrm{HuS}, 442$.

$14 \mathrm{HuS}, 445$.

15 "Secreto" no por "incomprensible", sino en el sentido de un conocimiento al que es sumamente difícil acceder, o al que sólo unos pocos pueden acceder (especulativamente). "Providencial" en tanto en cuanto se trata de una suerte de (divina) disposición anticipada que conduce al logro de un fin. Efectivamente, en la introducción a sus lecciones sobre filosofía de la historia, Hegel se había referido a ésta como Theodizeee: justificación de Dios (cf. Hegel, G. W. F., Lecciones sobre la filosofía de la historia universal, p. 56). La observación es sumamente importante para Rosenzweig, que en 1910 escribía: "Para Hegel la historia era divina, "teodicea" [...] Para nosotros, la religión es la "única y verdadera teodicea". - La lucha contra la historia en el sentido del siglo XIX es para nosotros, por ello, al mismo tiempo, lucha por la religión en el sentido del siglo XX" (GSI, 1, 112-113).

${ }^{16} \mathrm{HuS}$, 453. Subrayado mío. 
Estado, ni a través de una estatalización de la Iglesia, sino mediante una mundanización del Espíritu que es al mismo tiempo su más plena realización. Su condición de posibilidad viene dada por la separación institucional de Iglesia y Estado, propiciada a su vez por la separación interna de la Iglesia, esto es, por la Reforma. Sólo tras ésta fue posible que el Estado alcanzara la eticidad y la racionalidad autoconscientes: su determinación más plena.

O su destino: catolicismo y Estado racional o ético son en Hegel resueltamente incompatibles. La reconciliación de la realidad con el Espíritu, y del Estado con la conciencia religiosa, se produce en Hegel como resultado, según Rosenzweig, de una absolutización (histórica) del protestantismo -por el lado de la religión- y del Estado (prusiano) -por el lado de la política-. No hay mayor testimonio de esto, añade Rosenzweig, que el particular Rektoratsrede de Hegel, pronunciado con ocasión del tercer centenario de la Confessio Augustana: el comienzo de la época cuyo final, visto retrospectivamente, no tuvo su inicio en tiempos del filósofo suabo, sino en la década de esplendor del Canciller de Hierro. En lo que respecta al Hegel de Rosenzweig resta por tanto atender a su exposición del desarrollo de los pasos nacionales alemanes entre 1831, fecha de la muerte de Hegel, y 1871, año de la proclamación del Reich bismarckiano que, en nombre de la nación alemana, acabó convirtiendo la patria de Goethe, y con ella toda Europa, en una auténtica escombrera. La política compulsivamente expansionista implementada por el realismo político de cuño bismarckiano precipitó el final del dinamismo histórico, impulsado por la Reforma, al que dieron carta de naturaleza jurídica lo firmado en Westfalia (1648) y Utrecht (1713). Para Rosenzweig, a quien una muerte temprana evitó vivir la noche de la Shoá, lo acontecido entre 1914 y 1918 significó un final: el final de la idea nacional formada en el siglo XIX, heredera de la idea democrática y universalista de la revolución, e injertada cual retoño en la cepa de la vieja realidad de los Estados del siglo XVIII. Veamos qué papel otorga Rosenzweig a Hegel en este proceso de liquidación de toda una edad del mundo: la época del Estado nacional y del correspondiente Ius publicum europaeum.

III. 
Desde el principio mismo de las conclusiones del Hegel, Rosenzweig va a anunciar, o a denunciar más bien, la razón por la que la filosofía hegeliana ha sido considerada responsable de la catástrofe de Alemania: "Llegamos al fin. La intensidad de este fin ya la presentimos hoy, cuando ha colapsado el siglo de Bismarck, ante cuyas puertas está la vida de Hegel, tal como el pensamiento antecede a la acción." 17 La posición de Rosenzweig a este respecto es, no obstante, ambigua: Hegel no guió el íntegro desarrollo del siglo XIX. Más bien sólo abrió el camino. El universalismo de Hegel no conducía desde luego, a ojos de Rosenzweig y a diferencia de Meinecke, hacia una reducción de la autoridad del Estado, reducido a mero instrumento del Weltgeist. Al contrario, la metafísica a la que está ligada la idea de Estado en Hegel implicaba, según Rosenzweig, su fortalecimiento: la justificación del sacrosanto egoísmo estatal mediante su inserción en una filosofía de la historia que es más bien una teodicea. Pero "estatal" no significa necesariamente "nacional", es decir, "estatal-nacional". Lo que separa a Hegel del final del XIX es lo que separa a su Estado respecto de la idea de Estado nacional. Ésta fue introducida en Alemania, señala Rosenzweig, post-Hegelmortuum: por medio de los tratados sobre la naturaleza del Estado publicados por Dahlmann, Stahl y, sobre todo, Treitschke -todos ellos rankeanos y, por tanto, no hegelianos, por no decir anti-hegelianos $-{ }^{18}$. De modo muy conciso, cabría resumir las conclusiones del Hegel de Rosenzweig con las siguientes palabras: lo que la nación fue para el Estado pensado por Treitschke y realizado por Bismarck, eso mismo fue la voluntad del individuo para el Estado ideado por el liberal-estatal, no liberal-nacional, que había sido Hegel. Sólo negando la voluntad del individuo, según Rosenzweig, pudo la filosofía política alemana posterior a Hegel alumbrar la idea de un cuerpo estatal cuyo contenido viniese dado, única y exclusivamente, por la nación. Tal es la distancia entre Hegel y Treitschke, heraldo de Bismarck. También, no obstante, aquello que les une y a partir de lo cual cabe, a juicio de

${ }^{17} \mathrm{HuS}, 526$ [ed. cast.: "Hegel y el Estado. Conclusiones", en Lo bumano, lo divino y lo mundano: escritos, tr. M. G. Burello, Lilmod-Araucaria, Buenos Aires, 2007, p. 75].

${ }^{18}$ Cf. Dahlmann, F. C. , Die Politik, auf den Grund und das Maß der gegebenen Zustände zurückgeführt, Dieterich, Göttingen, 1835; Stahl, F. J., Die Pbilosopbie des Rechts nach geschichtlicher Ansicht, Mohr, Heidelberg, 1830-1837, I-III; y Treitschke, H. v., Politik. Vorlesungen gehalten an der Universität zu Berlin, Hirzel, Leipzig, 1897-1898, I-II. 
Rosenzweig, denunciar a Hegel como responsable intelectual, siquiera parcialmente, de la catástrofe alemana en la Primera Guerra Mundial ${ }^{19}$.

En el camino que va de la muerte del filósofo de Stuttgart a la fundación, clam, vi et precario, del Estado bismarckiano, Hegel aparece "como un filósofo de la historia, a lo sumo, pero no como un filósofo del Estado". ${ }^{20} \mathrm{Si}$ Hegel no había podido seguir el desarrollo nacional alemán, éste habría recurrido, conforme a la exposición de Rosenzweig, a la Weltgeschichte hegeliana, en su articulación con la filosofía política, para sacralizar el egoísmo y la estrechez, tanto interior como exterior, de la obra del Canciller de Hierro. En el momento en el que Rosenzweig establece este diagnóstico -1920- se levantaba sobre ella, sobre el Reich, un "campo de ruinas". No era sin embargo una catástrofe definitiva, pues no encontramos en Rosenzweig una consideración de la historia conforme al modelo -anti-mesiánico, en el sentido originario del término mesías, no en el cristiano-secularizado- de un despliegue progresivo mediante el cual son reabsorbidas las diferencias entre las naciones, sino como una multiplicidad de catástrofes ante la cual sólo tenemos derecho a decir, no que constituya una serie infinita, sino que desconocemos el día y la hora de la última: el instante en que acaecerá el final de los tiempos ${ }^{21}$. Y que su llegada, más bien su venida, el acaecimiento de la eternidad, y por tanto su temporalización, no puede ser retenida. Se trata, antes bien, de anticiparla prolépticamente: mesiánicamente. A ello se refiere la nueva secularización de la que habla Rosenzweig: aquello en lo que se cifra lo que La Estrella tiene de lucha por la religión y contra la historia -concebida begelianamente-. ${ }^{2}$

${ }^{19}$ Cf. HuS, 529-530 [ed. cast.: "Hegel y el Estado. Conclusiones", op. cit., p. 79]. Sobre el "momento hegeliano", vése no obstante Losurdo, D., Hegel y la catástrofe alemana, tr. A. García Mayo, Escolar y Mayo, Madrid, 2012, pp. 9-40, así como Duque, F., La Restauración. La escuela hegeliana y sus adversarios, Akal, Madrid, 1999. Quisiera dejar constancia de mi agradecimiento al profesor Félix Duque, quien tuvo la gentileza de leer y comentar con quien escribe una versión inicial del presente trabajo.

${ }^{20} \mathrm{HuS}, 529$ [ed. cast.: "Hegel y el Estado. Conclusiones", op. cit., p. 79]. El subrayado es mío.

${ }^{21}$ Cf. Rosenzweig, F., "Paralipomena”, op. cit., pp. 246 ss.

22 Cf. supra, n. 14, así como GSI, 2, 889-890. 
Ya advertimos, no obstante, que el presente análisis iba a ceñirse a lo que habríamos de llamar entonces "vieja secularización", esto es, la secularización cristiana del mesianismo judío, o la política mesiánica que Rosenzweig, en su enfrentamiento con la tiranía, denuncia como culpable de la catástrofe: último crimen de la serie de asesinatos, astutamente justificada, que es la historia hegeliana. Ante ella, al menos en un principio -al principio de La Estrella-, el hombre sólo puede hacer una cosa: gritar, pero no desesperadamente, o al menos sólo con una desesperación meramente aparente, pues en ello le va la vida, esto es, precisamente el contenido mismo de su esperanza, por no decir de toda esperanza, que Rosenzweig se niega a identificar con -ismo alguno.Veamos por tanto cuál es la relación que Rosenzweig establece en La Estrella entre historia y mesianismo o, mejor, entre historia y cristianismo en tanto que mundialización y universalización "mundialatinización", al menos en un primer momento, "globalización" posteriormente, con toda seguridad- de la elección mesiánica de Israel por medio de la idea nacional. Atendamos, en definitiva, a la historia exterior de la revelación (cristiana) cuya exposición toma el autor de La Estrella, ya no de Hegel, sino de las ultimísimas lecciones, sobre la Filosofía de la revelación, de su más enconado enemigo: F. W. J. Schelling23.

El 30 de noviembre de 1916, en carta a su interlocutor por excelencia (Eugen Rosenstock), Rosenzweig se refiere a estas postreras Vorlesungen schellinguianas y a la distinción entre tres épocas del cristianismo -cada una bajo el signo de un apóstol: Pedro, Pablo y Juan-, que cabe encontrar en ellas. ${ }^{24}$ Rosenzweig volverá a hacer uso de este esquema en la introducción al cuando menos peculiar tratado teológico-político que ofrece a sus lectores el tercer libro de La Estrella, sintomáticamente encabezado por el lema -más una amenaza que una dedicatoria-: In Tyrannos!, esto es, “iContra los tiranos!”, tanto del Reino de los Cielos como de este mundo -si es que ambos pueden

${ }^{23}$ Cf. Schelling, Philosophie II, 293, así como Fricke, M., Franz Rosenzweigs Philosophie der Offenbarung. Eine Interpretation des Sterns der Erlösung, Königshausen-Neumann, Würzburg, 2003, p. 273.

${ }^{24}$ Cf. GSI, 1, 302ss., así como Mosès, S., El ángel de la historia. Rosenzuveig, Benjamin, Scholem, tr. A. Martorell, Cátedra, Madrid, 1997, p. 47. 
verdaderamente ser distinguidos ${ }^{25}-$. Ahora bien, la adopción del esquema trinitario schellinguiano (y joaquinita) está mediada por la profunda formación hegeliana de Rosenzweig y, en particular, por la teoría de la secularización elaborada por Hegel a partir de la articulación de filosofía política y filosofía de la historia, así como de la contraposición sistemática de los roles desempeñados por Estado e Iglesia -religión o comunidad eclesiástica- en este contexto. Como hemos visto, no es el caso que esta teoría de la secularización fuese para Rosenzweig simplemente un objeto más de estudio. Constituye el centro mismo de las preocupaciones de Rosenzweig en lo que se refiere a Hegel (y el Estado). La historia del cristianismo presentada por Rosenzweig a partir del esquema schellinguiano no deja intacto a éste sino que, por así decir, lo hegelianiza: lo emplea para exponer una teoría de la secularización que ha de ser calificada de hegeliana.

La primera alusión de Rosenzweig al problema de la secularización en su opus magnum hace alusión a una "mundanización del mundo" (Verweltlichung der Welt) cuyo origen es localizado por el autor de La Estrella en el surgimiento del protestantismo. Se trata, en opinión de Rosenzweig, de la característica fundamental de lo que, por oposición a la época petrina de la Iglesia, es decir del Populus Christianus cuyo centro de gravedad se situaba en Roma y su catolicismo, el filósofo de Kassel denomina "los siglos paulinos". ${ }^{26}$ La peculiaridad inherente a la iglesia paulina no es otra, según Rosenzweig, que la separación tajante entre lo espiritual y lo mundano: "La mutua protesta de la fe contra el mundo y del mundo contra la fe". ${ }^{27}$ Esta escisión radical permitió al protestantismo la realización de aquello a cuya consecución estaba llamado: la cristianización del alma gentil que, aferrado a la exterioridad, el catolicismo había dejado escapar. Ahora bien, la Iglesia paulina, siendo invisible por principio -esto es: no siendo de este mundo-, necesita de su visibilización en el mundo como institución jurídica independiente del Estado y, de hecho, necesariamente enfrentada al Estado. ${ }^{28}$

${ }^{25}$ Cf. EdR, 326, así como Casper, B., "Der Andere, der Dritte und die Bürgschaft für die Gerechtigkeit", op. cit., p. 190.

${ }^{26}$ Cf. EdR, 336-337.

${ }^{27} \mathrm{EdR}, 337$.

${ }^{28}$ Cf. EdR, 456. 
A la mundanización del mundo corresponde inevitablemente, en estricta aplicación de la ruptura entre Geist y Welt sobre la propia Iglesia, una "mundanización del cristianismo" (Verwelttichung des Christentums). La escisión que el catolicismo contenía in nuce -una escisión entre dos caminos, es decir dos órdenes, que no lo son sino de una unidad más amplia: la Respublica Christiana - es consumada por el protestantismo, que en este sentido -como en Schelling- no hace sino sacar a la luz la verdad misma del catolicismo, al cual la historia habría por tanto despachado definitivamente. Debe acontecer aún, no obstante, la reconciliación de fe y mundo, de Iglesia y Estado. Tal es el evangelio de Hegel, como de Schelling -y tantos otros-. Con una diferencia: Hegel concibió su propia época como la del parto y la transición hacia la nueva aetas; Schelling en cambio, como Fichte, postergó sine die la reconciliación de Dios y mundo. Sólo Hegel habría tenido, según Rosenzweig, que sigue en este punto la interpretación meineckeana del filósofo suabo, el atrevimiento de afirmar la efectiva realización del Espíritu en el mundo, de afirmar la (supuesta) ${ }^{29}$ identidad de realidad y racionalidad: el advenimiento de la Iglesia joánica, o la consumación del cristianismo, esto es, la cristianización del destino del mundo, y de sus pueblos, a través del establecimiento de la política mesiánica (el nacionalismo) como forma de la civilización occidental.

La aparición de la iglesia joánica en el mundo significa así, según Rosenzweig, una cristianización plena de los pueblos que componen la civilización occidental; es decir, una secularización total del cristianismo, en el sentido de una identificación del destino de la cristiandad con el destino del saeculum: una cristianización de la vida histórica de los pueblos del mundo que se hace patente a través del auge del nacionalismo, fenómeno determinante del devenir histórico desde 1800 -en realidad, desde 1789: la Revolución Francesa- y hasta la Gran Guerra -propiamente, al menos hasta la Segunda Guerra Mundial, que Rosenzweig, tempranamente malogrado a causa de una terrible enfermedad, no llegó a vivir y, sin embargo, fue capaz de vislumbrar, por ejemplo, en las páginas finales de Globus ${ }^{30}-$. A esta interpretación de la

${ }^{29}$ Cf. supra, nt. 10.

${ }^{30}$ Cf. Rosenzweig, F., "Globus. Studien zur weltgeschichtlichen Raumlehre”, en Der Mensch und sein Werk. Gesammelte Schriften (III). Zweistromland. Kleinere Schriften zu Glauben und Denken, Martinus Nijhoff, Haag, 1984, pp. 366ss. 
secularización están consagradas las páginas dedicadas por Rosenzweig a la política mesiánica de los pueblos del mundo. El término "secularización", sin embargo, no hace acto de presencia en estas líneas de La Estrella. Es decisivo, en cambio, en una carta fechada por Rosenzweig el 7 de noviembre de 1916.31

La misiva, dirigida a Eugen Rosenstock, comienza con una breve disquisición sobre la importancia del hecho de que el término griego ethne, es decir "paganos" (Heiden), en el sentido de "pueblos" (Völker)-como el correspondiente Ioudaioi, es decir "judíos", Juden-, no tuviese en su origen forma alguna terminada en -ismos, en el sentido del sufijo alemán -tum, que denota condición, naturaleza, cuando no idea, concepto (Begriff). ${ }^{32}$ No obstante su aparente trivialidad, esta digresión de Rosenzweig está dotada de un enorme significado, toda vez que la interpretación rosenzweiguiana de lo genuinamente mesiánico -a diferencia del mesianismo secularizado como nacionalismo imperialista- se refiere a una forma de fe en un Mesías que supera todo "-ismo" particular, ya sea el judaísmo, el cristianismo o el propio mesianismo, quizá laico-utópico. ${ }^{33}$ Paradójicamente, continúa Rosenzweig, ethnismos, el particular -ismos correspondiente al griego ethne, habría surgido, no como creación de la idea de paganismo (Heidentum), sino como resultado de un proceso de cristianización (y latinización) del concepto de "pueblo", es decir como "nacionalismo" (Nationalismus): cada pueblo sabe y cree que, a través de la historia, de su historia, de la historia que él hace, se dirige hacia Dios. $^{34}$

Cada pueblo se sabe el pueblo elegido. O cree serlo. Su política se convierte en política mesiánica: el nacionalismo moderno es, por lo tanto, el resultado de una secularización de los conceptos de mesianismo y elección, previamente universalizados por el cristianismo -frente a su originario carácter particular, propio del que se considera el único pueblo elegido: el eterno pueblo de sacerdotes-. "Secularización" en el sentido de que, frente al

${ }^{31}$ Cf. GSI, 1, 280-289.

32 Cf. GSI, 1, 281.

33 Cf. EdR, 493, así como García-Baró, M., La compasión y la catástrofe. Ensayos de pensamiento judio, Sígueme, Salamanca, 2007, p. 243.

34 Cf. GSI, 1, 281. 
mesianismo judío, este mesianismo secularizado que irrumpe en la historia como nacionalismo ha de realizarse necesariamente en y por la historia. La elección de los pueblos, su carácter mesiánico, consiste en que las naciones se sienten protagonistas de la historia, portadoras de la Weltgeschichte: el nacionalismo se exalta y, dotado de una voluntad supranacional y orientada al mundo (como la del cristianismo), deviene imperialismo a partir de una secularización, esta vez, de la idea cristiana de misión, entendida ya no como misión evangelizadora, sino como misión civilizadora. ${ }^{35}$ Es por tanto la recuperación de la idea de misión por parte de las iglesias reformadas la que, según Rosenzweig, habría sentado las bases del cristianismo del futuro, es decir de la era joánica: la cristianización consumada de la civilización occidental en cuyos miembros, dotados ahora de una voluntad que va más allá de sí mismos, de su propio derecho (una voluntad supranacional), se secularizan las ideas de elección y de mesianismo. ${ }^{36}$

La civitas Dei deviene civitas terrena o, mejor, la civitas terrena pretende ser civitas Dei, iglesia en la que autoconservación (salus) y fidelidad a lo más alto (fides) - en este caso la historia universal en tanto que perspectiva suprema bajo la cual se comprenden los pueblos, todos ellos pretendidamente elegidos- son indiscernibles: la secularización del cristianismo consumada en la era joánica conduce a la irrupción de una política que, en cuanto mesiánica, quiere atribuir la forma del Reino de Dios a la realidad histórica. La cristianización del concepto de pueblo, el nacionalismo como expresión política de la secularización plena del cristianismo, conduce así a una sacralización de la política y de la historia que se hace patente, en primer lugar, en la santificación de la guerra. Ésta es concebida, a un mismo tiempo, como lucha tanto por la propia existencia (salus) como por el sentido del mundo (fides), es decir, como "guerra de religión". ${ }^{37}$ Esta expresión, empleada por Rosenzweig, debe entenderse en un sentido bien distinto al de las guerras confesionales europeas de comienzos de la Modernidad. No se trata de una lucha por confesión religiosa alguna, despolitizadas todas a lo largo de los siglos paulinos, sino de que el Estado y su papel en la historia han devenido, ellos mismos, realidades sacrosantas: la voluntad de Dios -es decir, ahora, el

35 Cf. GSI, 1, 283, así como EdR, 390.

36 Cf. EdR, 337.

$37 \mathrm{EdR}, 392$. 
sentido de la historia- se realiza en la suerte que los Estados, al margen de toda confesión religiosa particular, corren en la guerra. Si Rosenzweig había dicho que su interpretación de la "política mesiánica" no era otra cosa que una "teoría de la guerra" 38 , ésta no es sino el corolario de una "teoría de la secularización" -más bien de la mundanización- que, no obstante el tamiz schellinguiano, responde a una notoria y decisiva influencia hegeliana en el autor de La Estrella.

Recibido: 9/2014. Aprobado: 12/2014.

${ }^{38}$ Cf. Rosenzweig, F., "El nuevo pensamiento. Observaciones adicionales a La Estrella de la Redención", en El nuevo pensamiento, tr. Á. E. Garrido Maturano, Adriana Hidalgo, Buenos Aires, 2005, p. 43. 